\title{
The role of genetic testing in dyslipidaemia
}

\author{
Amanda J. Berberich, Robert A. Hegele \\ Departments of Medicine and Biochemistry, and Robarts Research Institute, Schulich School \\ of Medicine and Dentistry, Western University, London, Ontario, Canada
}

\begin{abstract}
Summary
Dyslipidaemias encompass about two dozen relatively rare monogenic disorders and syndromes for which the genetic basis has largely been defined. In addition, the complex polygenic basis of disturbed lipids and lipoproteins has been characterised in many patients, and has been shown to result from accumulation of many common polymorphisms with small effects on lipids. Genetic technologies, including dedicated genotyping and sequencing methods can detect both rare and common DNA variants underlying dyslipidaemias. Some dyslipidaemias may be clinically silent for years, but early diagnosis, including genetic diagnosis, may permit early intervention to prevent or delay deleterious downstream clinical consequences, such as premature vascular disease or acute pancreatitis. The potential clinical utility of genetic testing for familial hypercholesterolaemia, familial chylomicronaemia syndrome, lysosomal acid lipase deficiency and some others will increase demand for reliable genetic diagnostic methods. We review some current technologies, such as targeted next-generation sequencing that seem to be helpful with DNA diagnosis of dyslipidaemias. We also address technical, biological and clinical limitations of genetic testing in dyslipidaemias. Finally, genetic counselling issues, the potential impact of results on patients and health care providers, current gaps and future directions will be discussed.
\end{abstract}

Key words: Genetic testing; dyslipidaemia; familial hypercholesterolaemia; familial chylomicronaemia.

Received 26 September, revised 8 October, accepted 10 October 2018 Available online 14 December 2018

\section{INTRODUCTION}

Increased appreciation of genetics by clinicians, researchers and the general public parallels the recognition of its causative role in many diseases. Genetic understanding is wellestablished for dyslipidaemias, a collection of $\sim 25$ named metabolic disorders that are characterised by extreme serum levels of lipoprotein particles such as high-, low- and intermediate-density lipoproteins (HDL, LDL and IDL, respectively) and triglycerides (TG). Inherited differences underlie both variation in lipid phenotypes in the general population and susceptibility to dyslipidaemias in patients. Furthermore, the often complex and multifactorial nature of these clinically relevant traits is becoming more evident.
The archetypal condition in which genetic analysis is often being applied clinically is familial hypercholesterolaemia (FH). FH is a common inherited disorder, with population prevalence of $\sim 1$ in $250 .{ }^{1} \mathrm{FH}$ predisposes to premature development of plaques in the coronary, central and peripheral vasculature that can lead to early-onset atherosclerotic cardiovascular disease (ASCVD), including coronary heart disease, stroke and limb ischaemia. ${ }^{1}$

Another familial dyslipidaemia is severe hypertriglyceridaemia, which can lead to acute pancreatitis that can be life-threatening. ${ }^{2}$ Genetic analysis may be helpful in certain cases of severe hypertriglyceridaemia, such as familial chylomicronaemia syndrome (FCS). Molecular defects in other pathways involved in lipoprotein metabolism can have similarly clinically relevant effects for which genetic analysis can provide clinically actionable data.

Rapidly declining costs make it possible to consider genetic testing in selected dyslipidaemic patients or their asymptomatic relatives. However, precise indications for genetic testing for most dyslipidaemias have not yet been clearly established and there are currently no clinical guidelines for genetic testing in dyslipidaemia. We explore some issues surrounding genetic testing in dyslipidaemia, endeavouring to provide some guidance to practitioners on its role and utility in the clinical setting.

\section{GENETIC TESTING}

'Genetic testing' refers to several methods used to determine the genotype of an individual. A key factor in selecting the type of testing and technology is whether the patient is suspected to have a rare mutation of large clinical effect causing a monogenic dyslipidaemia ${ }^{2}$ versus a collection of common single nucleotide polymorphisms (SNPs) with individually small effects that collectively create susceptibility to dyslipidaemia under a polygenic model of inheritance. $^{3}$ There are several different methods in current clinical use $^{4}$ (Table 1).

\section{Sanger sequencing}

Automated Sanger or dideoxy chain termination sequencing is used to examine small DNA segments, usually a single gene or even a single exon. It can precisely detect a single nucleotide change, often a heterozygous rare variant with a large clinical effect, in a known gene causing a monogenic disorder (we use the terms 'mutation' and 'large effect rare variant' interchangeably). This method can be applied in family cascade screening to follow the inheritance of a 
Table 1 Genetic testing methods

\begin{tabular}{|c|c|c|c|c|c|c|c|c|}
\hline \multirow[t]{2}{*}{ Method } & \multirow[t]{2}{*}{ Description } & \multirow[t]{2}{*}{ Strengths } & \multirow[t]{2}{*}{ Weaknesses } & \multicolumn{4}{|c|}{ Types of detectable variation } & \multirow[t]{2}{*}{ Best use } \\
\hline & & & & $\mathrm{CNV}$ & Rare variants & $\begin{array}{l}\text { Common } \\
\text { variants }\end{array}$ & PRS & \\
\hline $\begin{array}{l}\text { Sanger } \\
\text { sequencing }\end{array}$ & $\begin{array}{l}\text { Amplification using individual } \\
\text { primer pairs in separate } \\
\text { amplification and } \\
\text { sequencing reactions }\end{array}$ & $\begin{array}{l}\text { - Gold standard for diagnosis } \\
\text { of small DNA changes } \\
\text { - Highest depth of coverage }\end{array}$ & $\begin{array}{l}\text { - Labour intensive } \\
\text { - Highest cost when done in } \\
\text { high volumes }\end{array}$ & No & Yes & No & No & $\begin{array}{l}\text { - Single gene tests } \\
\text { - Confirmation of presence/ } \\
\text { absence of a known } \\
\text { mutation in familial cascade } \\
\text { testing }\end{array}$ \\
\hline $\begin{array}{l}\text { Targeted NG } \\
\text { sequencing }\end{array}$ & $\begin{array}{l}\text { Simultaneous screen of a pre- } \\
\text { selected subset of genes }\end{array}$ & $\begin{array}{l}\text { - Maximise diagnostic yield } \\
\text { and minimise off-target } \\
\text { results } \\
\text { - High depth of coverage }\end{array}$ & $\begin{array}{l}\text { - Will not find mutations in } \\
\text { genes outside the panel } \\
\text { design }\end{array}$ & $\mathrm{Yes}^{\mathrm{a}}$ & Yes & Yes $^{\mathrm{b}}$ & Yes $^{\mathrm{b}}$ & $\begin{array}{l}\text { - Investigating a condition with } \\
\text { multiple causal/contributing } \\
\text { genes }\end{array}$ \\
\hline $\begin{array}{l}\text { Whole exome } \\
\text { sequencing }\end{array}$ & $\begin{array}{l}\text { Simultaneous screen of all } \\
\text { exons (i.e., coding } \\
\text { sequences) in an individual } \\
\text { ( } 2 \% \text { of total DNA) }\end{array}$ & $\begin{array}{l}\text { - Can identify new or } \\
\text { unexpected genes } \\
\text { - Could be re-examined for } \\
\text { other conditions at a later } \\
\text { date }\end{array}$ & $\begin{array}{l}\text { - Potential for off-target results } \\
\text { - Higher probability of } \\
\text { uncertain results }\end{array}$ & $\operatorname{Yes}^{\mathrm{a}}$ & Yes & No & No & $\begin{array}{l}\text { - Conditions with an unclear } \\
\text { genetic basis } \\
\text { - Conditions expected to be } \\
\text { genetic but without a cause } \\
\text { identified on other testing }\end{array}$ \\
\hline $\begin{array}{l}\text { Whole genome } \\
\text { sequencing }\end{array}$ & $\begin{array}{l}\text { Simultaneous screen of all } \\
\text { coding and noncoding DNA } \\
\text { in an individual, including } \\
\text { mitochondrial }\end{array}$ & $\begin{array}{l}\text { - Includes all genetic material, } \\
\text { including regulatory regions } \\
\text { - Could be re-examined for } \\
\text { other conditions at a later } \\
\text { date }\end{array}$ & $\begin{array}{l}\text { - Lower depth of coverage } \\
\text { - Higher probably of uncertain } \\
\text { and off target results } \\
\text { - Labour intensive }\end{array}$ & Yes & Yes & Yes & Yes & $\begin{array}{l}\text { - Same as for whole exome but } \\
\text { provides more } \\
\text { comprehensive data }\end{array}$ \\
\hline Genotyping & $\begin{array}{l}\text { Rapid screen of entire genome } \\
\text { using an array of common } \\
\text { variant SNPs }\end{array}$ & $\begin{array}{l}\text { - Rapid } \\
\text { - Lower cost } \\
\text { - Entire genome examined }\end{array}$ & $\begin{array}{l}\text { - Cannot detect novel variants } \\
\text { - No sequence-level data }\end{array}$ & Yes & Yes $^{\mathrm{b}}$ & Yes $^{\mathrm{b}}$ & Yes & $\begin{array}{l}\text { - Can be used to look for } \\
\text { disease risk, ethnicity and to } \\
\text { determine familial } \\
\text { relationships }\end{array}$ \\
\hline $\begin{array}{l}\text { Polygenic risk } \\
\text { score }\end{array}$ & $\begin{array}{l}\text { Derived by summing the minor } \\
\text { effects of several common } \\
\text { variants to generate an } \\
\text { overall estimate of risk }\end{array}$ & $\begin{array}{l}\text { - Can provide information on } \\
\text { risk that will not be apparent } \\
\text { with other forms of } \\
\text { sequencing }\end{array}$ & $\begin{array}{l}\text { - Ethnic variations can limit } \\
\text { use } \\
\text { - Predictive value varies with } \\
\text { each score }\end{array}$ & N/A & N/A & N/A & N/A & $\begin{array}{l}\text { - Used as a complement to } \\
\text { traditional sequencing to } \\
\text { help provide an additional } \\
\text { clinical prediction of risk }\end{array}$ \\
\hline
\end{tabular}

CNV, copy number variant; PRS, polygenic risk score.

${ }^{a}$ With specialised bioinformatics tools.

${ }^{\mathrm{b}}$ If designed to target. 
previously identified mutation. Sanger sequencing has the added benefit of providing only the desired genetic result with little to no chance of incidental findings. The cost of Sanger sequencing varies depending on the size and number of genes and exons tested, with each 1-2 kilobase length of DNA requiring: (1) design and purchase of a primer pair ( $\$ 30$ USD); and (2) polymerase chain reaction (PCR)-based bi-directional sequencing ( $\$ 20$ USD). Both in the clinic and research lab, Sanger sequencing often serves as the 'gold standard' confirmation when a potentially causative variant is found with other methods. 5,6

\section{Next-generation sequencing}

Next-generation sequencing (NGS) refers to various methods that each use a massively parallel sequencing design to amplify and examine multiple segments of DNA concurrently. ${ }^{7}$ This technique is typically applied to detect a rare causative variant for a monogenic condition when many possible mutations exist, and can be used to sequence: (1) a targeted selection of pre-specified genes; (2) all expressed protein-coding sequences ('whole exome sequencing' or WES) representing $2 \%$ of the entire genome; or (3) all coding and non-coding regions comprising the entire genome ('whole genome sequencing' or WGS). ${ }^{7,8} \mathrm{WES}$ is optimal for detecting rare coding variants primarily, while WGS detects all common and rare variants in all coding and non-coding regions, producing an enormous data file for each sample.

Targeted NGS panels can be designed to both screen for rare variants in coding regions and concurrently evaluate non-coding common SNPs as part of a polygenic risk score. Several targeted panels have been clinically validated and their cost is decreasing steadily. ${ }^{9}$ The cost of this technology varies depending on how many genes are concurrently assessed, with approximate costs per sample of $\$ 300$ for a targeted panel, \$800-1200 for WES and \$3-10,000 for WGS. ${ }^{8}$ For genetic analysis of dyslipidaemias, targeted sequencing panels are the current standard for clinical diagnosis. $2,10-12$

\section{Genotyping}

Genotyping refers to various types of dedicated, inexpensive methods to directly assay specific known rare variants or common SNPs. Many different technical and chemical platforms can be used for dedicated genotyping. Depending on the indication, these methods can evaluate a single or numerous pre-defined SNPs already known to be associated with certain dyslipidaemias. A familiar example would be genotyping of $A P O E$ isoforms to identify E2/E2 homozygosity in suspected type 3 hyperlipoproteinaemia (dysbetalipoproteinaemia).

Large-scale extensive genotyping employs high density genome-wide microarrays in unbiased genome-wide association studies (GWAS) to discover associations between millions of SNP markers and either quantitative or qualitative complex clinical traits. ${ }^{7}$ Once a subset of SNP genotypes has been definitely associated with clinical phenotypes, a smaller, less costly panel can be constructed using dedicated chemistry (e.g., TaqMan), mass spectrometry or custom-made microarrays, allowing focused testing of clinical samples. Genotyping methods detect only those DNA changes they have been designed to capture; ${ }^{7}$ however, for clinical applications, there is no need to screen for every possible base pair variation or to detect a rare unknown mutation. Many commercial direct-to-consumer DNA tests employ SNP genotyping.

\section{Polygenic risk scores using SNPs}

Dyslipidaemia sometimes results from accumulation of multiple small-effect DNA variations. These common allelic variants each only slightly affect lipid levels, but when present in sufficient numbers in an individual's genome they can underlie a phenotype resembling that of an individual carrying a single, rare, large-effect mutation. ${ }^{13-15}$ For example, several SNPs scattered throughout the genome have been shown in GWAS to have modest but highly reproducible effects on lipid traits, e.g., HDL cholesterol (C), LDL-C or TG. ${ }^{3}$ Most people have some SNP alleles that raise and others that lower a lipoprotein trait, resulting in an average serum level. However, some individuals, by unlucky chance, inherit an excess of SNP alleles that concertedly alter the lipid trait in the same direction (e.g., they all raise LDL-C). ${ }^{16}$ High cholesterol from accumulation of many small-effect SNPs can be indistinguishable clinically from a single gene rare variant cause. ${ }^{17}$ However, this mechanism of disease is not detected by some forms of genetic testing, e.g., WES or targeted sequencing that is not designed to concurrently detect common associated non-coding SNPs. ${ }^{16,17}$ These lipid trait-altering SNP genotypes can be assessed using dedicated allele-specific detection methods, such as TaqMan-based assays or SNP microarrays, or by designing targeted NGS panels to detect them.

Once these SNPs have been genotyped, they are entered into a scoring scheme to quantify their cumulative effect on the lipid trait. These scores sum the expected effect of each individual SNP in a given patient, yielding a polygenic SNP score (also called a 'polygenic risk score' or 'polygenic trait score') that can be compared to a normolipidaemic population. An individual with a high polygenic score, e.g., $>90$ th percentile for the population, would be predisposed to develop clinically apparent dyslipidaemia. ${ }^{16,17}$

Since targeted NGS is the method of choice to detect rare mutations, a portion of excess capacity on the panel could be designed to concurrently sequence non-coding regions harbouring key SNPs needed for a polygenic risk score. This means that both rare and common DNA variation underlying clinical dyslipidaemia can be concurrently evaluated using a single laboratory method, which saves time, effort and expense. ${ }^{2}$ Using NGS to generate polygenic risk scores adds information on the underlying genetic contribution to a phenotype. ${ }^{2}$ We have shown that it fills in the missing genetic gap for many disorders, such as in FH, where up to one-third of clinically-ascertained cases without a discrete rare largeeffect mutation actually have a high polygenic score; ${ }^{17}$ similar findings are seen in patients with extreme levels of HDL-C. ${ }^{18}$

SNP scores have been generated using a small number of SNPs, several hundred SNPs and recently several million SNPs; ${ }^{15}$ the score's predictive value has been demonstrated to increase with the number of SNPs incorporated. There is no current consensus for how to use polygenic risk scores in dyslipidemia diagnosis. Since many dyslipidaemic patients have a polygenic rather than monogenic aetiology, polygenic 
risk scores could play an increasing role in genetic diagnosis, ${ }^{15}$ especially if they can be shown to predict clinical outcomes $^{15}$ or response to interventions.

\section{Copy number variation}

The term copy number variation $(\mathrm{CNV})$ refers to a specific quantitative type of genetic variant characterised by altered dosage of a genomic region, i.e., deviation from the usual two copies of a particular genomic sequence. CNVs can be largescale duplications or deletions that affect a whole exon, gene or even several genes. They are usually sub-chromosomal in size and can cumulatively affect up to $10 \%$ of the genome, compared to only $1-2 \%$ of total genomic DNA that varies due to all known SNPs and small-scale rare variants.

CNVs in the LDLR gene account for up to $10 \%$ of causal FH mutations, making it important to check for these in the diagnosis of $\mathrm{FH}^{19}$ CNVs are difficult to directly detect with DNA sequencing, especially large deletions, which eliminate one copy of normal sequence that cannot be amplified and chemically processed by Sanger methodology. In this instance, only the single normal allele is read, but there is no way to tell that the second allele is missing. Similarly, an extra copy of a long stretch of genomic DNA is difficult to detect by sequencing methods that are optimised to identify small-scale variation. In the past, separate methodology, such as high definition cytogenetic analysis, or comparative genomic hybridisation with DNA microarrays or multiplex ligation-dependent probe amplification (MLPA) was required. For diagnostic laboratories, establishing an additional method to detect CNVs increased costs: e.g., MLPA of the $L D L R$ gene costs $\sim \$ 100$ USD per sample on top of the sequencing cost.

Fortunately, new bioinformatic tools can detect CNVs from raw NGS data, without additional reagents. ${ }^{19}$ Targeted NGS can thus detect rare, large-effect, small-scale variants and CNVs causing monogenic dyslipidaemias, in addition to SNPs used in polygenic risk scores. The ability to simultaneously evaluate several forms of variation using a single laboratory method improves diagnostic yield (i.e., fewer false negatives), without increasing the cost.

\section{Other advanced genetic tests}

Other methods may provide additional useful clinical information for dyslipidaemias. These techniques include: (1) proteomic methods, whereby protein expression is assessed at the cell or tissue level to indirectly evaluate gene expression; (2) metabolomics, which refers to the concurrent assessment of small molecule metabolites from serum or tissue samples to indirectly evaluate in a targeted manner variations in gene products involved in intermediate biochemical pathways or in drug metabolism; ${ }^{20,21}$ and (3) genome-wide transcriptome analysis of RNA expression (e.g., RNASeq) that can be further used to assess gene expression and patterns of alternative RNA splicing within a cell or tissue. ${ }^{22}$

Epigenetics is increasingly recognised as affecting genotype-phenotype relationships. Incorporating epigenetic analysis using methylation arrays ${ }^{23}$ or other assays into standard genetic practice may be on the horizon. ${ }^{9}$ However, for dyslipidaemias this is limited by the fact that epigenetic imprinting in relevant tissues such as liver or intestine is not faithfully reflected by patterns in white blood cells, which are the usual convenient source of genetic material.

\section{Determining pathogenicity}

Even when a rare variant in a known causative gene for monogenic dyslipidaemia is detected, its causality or pathogenicity is not guaranteed. DNA changes may be synonymous, meaning that they code for the same amino acid as the base pair that was replaced, or if non-synonymous, they may still be benign, in that the altered amino acid has no clinically relevant impact on the structure or function of the translated protein.

The highest grade of evidence for a mutation's pathogenicity is a functional study performed in a research laboratory showing that the mutation causes a measurable change in an assay or model system. ${ }^{24}$ Familial segregation analysis that shows complete genotype-phenotype concordance is also helpful; i.e., each affected family member carries the variant, while unaffected members do not carry the variant. The evidence becomes statistically stronger as more family members are tested. Conversely, the presence of the mutation in healthy, phenotype-free individuals argues against pathogenicity. ${ }^{24}$

A second tier of evidence for pathogenicity comes from 'bioinformatics', i.e., computational modelling and analysis. Here, prediction software tools employ complex but validated mathematical algorithms to predict the impact of the amino acid change based on the degree of evolutionary conservation of the wild-type amino acid, the predicted 3-D structure of the resultant protein, and other circumstantial information. ${ }^{24}$ However, these methods sometimes give inconsistent results for the same mutation. Even after manual adjudication by human genetics experts, there can be disagreement regarding a mutation's potential pathogenicity. Nonetheless, trained, knowledgeable and skilled individuals must be available as back-up to interpret results of genetic tests, both to avoid incorrectly assigning pathogenicity to a benign change or incorrectly ascribing neutral function to a truly pathogenic variant.

\section{Potential benefits}

Genetic testing, in the best case, can provide a definitive diagnosis. Possible benefits include tailoring management to the individual patient (Table 2). For instance, heterozygous $\mathrm{FH}$ from $\mathrm{CNV}$ s seems to be a more severe phenotype than $\mathrm{FH}$ from single nucleotide variants. ${ }^{17,25}$ Genetic diagnosis could reduce delay in selecting an appropriate treatment, i.e., possibly more aggressive LDL-C lowering strategies. ${ }^{21}$ Additionally, for some monogenic dyslipidaemias, a genetic diagnosis is needed to secure funding for newer therapies. For instance, in certain jurisdictions, third party private coverage for injectable inhibitors of proprotein convertase subtilisin kexin 9 (PCSK9) for the treatment of $\mathrm{FH}$ requires a genetic diagnosis.

Some genetic dyslipidaemias also carry risk for other outcomes, such as hepatic cirrhosis in cholesteryl ester storage disease (CESD) or Wolman disease due to rare bi-allelic LIPA variants, ${ }^{26}$ where third party coverage to receive sebelipase (intravenous lysosomal acid lipase infusion) often depends on a positive DNA diagnosis. Other genetic conditions can mimic a classic dyslipidaemia but because they are 
Table 2 Clinically actionable impact of genetic diagnosis in selected monogenic dyslipidaemias

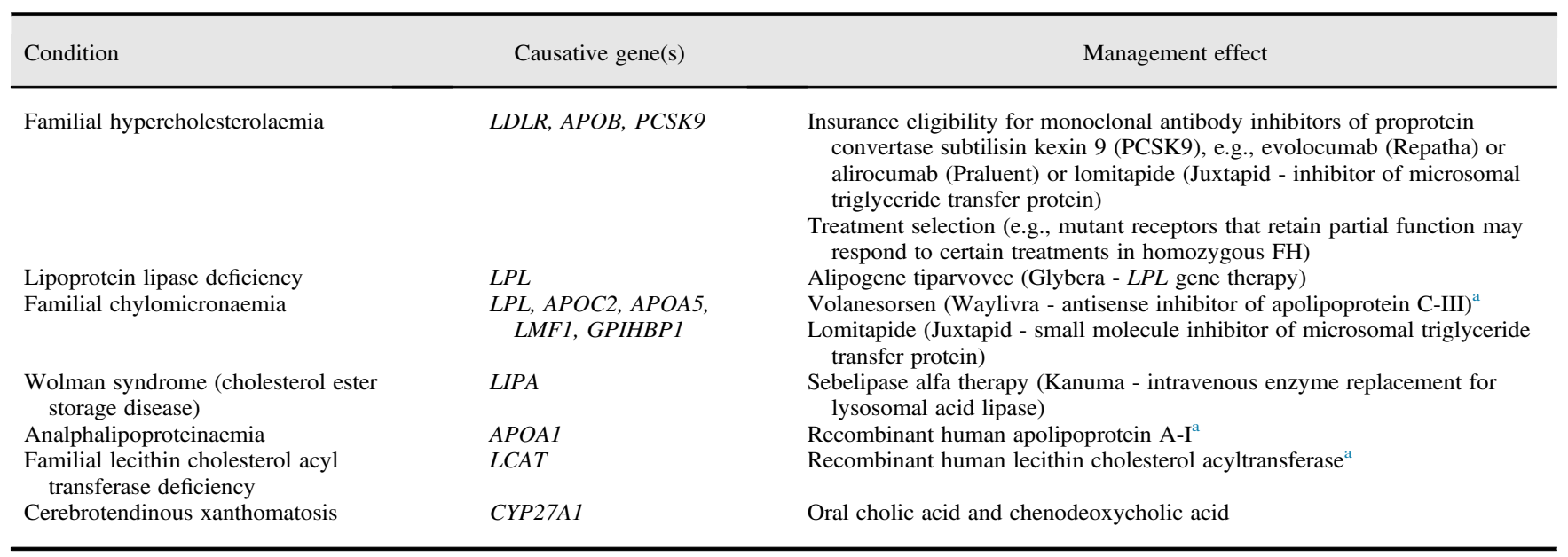



treated differently, a genetic diagnosis can be helpful. For instance, sitosterolaemia sometimes resembles $\mathrm{FH}$, but its management focuses on intestinal cholesterol absorption inhibition rather than statins. ${ }^{27}$

Furthermore, patients with some dyslipidaemias do not express overt symptoms, such as atherosclerosis, until later in the disease course. When early prevention and management have been shown to delay onset of complications, there is benefit to identify and treat these individuals assertively as early as possible, as with heterozygous $\mathrm{FH}^{28}$

Furthermore, opportunities may arise for early detection and intervention for children or family members of individuals with a discrete monogenic dyslipidaemia. For instance, heterozygous $\mathrm{FH}$ follows an autosomal dominant transmission pattern, meaning that half of all first-degree relatives also have the condition. ${ }^{29}$ Many $\mathrm{FH}$ individuals in the pre-clinical stage of ASCVD would benefit from an early diagnosis. A definite DNA diagnosis could also impact on family planning, especially for the parents of children presenting with severe homozygous dyslipidaemias; a similar presentation would be expected in onequarter of future offspring. There may also be benefits for adults who are considering starting a family and wish to evaluate potential risks, which can be explained by a genetic counsellor.

Potential non-clinical benefits of genetic testing include patient empowerment. Knowing their diagnosis, even without interventions or prevention strategies, can still provide awareness of the expected disease course, allowing for advanced planning, and emotional and mental control over healthcare. ${ }^{30}$ A negative test result for an at-risk individual may provide psychological relief and reduces costs of future surveillance. $^{3}$

Systematic genetic testing in an academic clinic or a disease registry can also improve understanding of the pathophysiology and consequences of a condition. This in turn can lead to the development of new, more effective pharmacological treatments or management strategies. A prime example is the development of PCSK9 inhibitors, which owe their inception and development to genetic studies of altered lipids in patients, families and communities.

\section{Potential drawbacks}

A major limitation to genetic testing is potentially prohibitive costs, especially for widespread WES or WGS. However, costs are dropping rapidly, as predicted by Moore's law of the economics of new technologies. ${ }^{9}$ The first completed whole DNA sequence in 2000 cost $\$ 2.7$ billion; the cost today is $\$ 3-10,000$ or less for a whole genome and much less for a whole exome. As costs continue to decline, this barrier is increasingly overcome.

Ethical dilemmas can arise from increased use of genetic testing. Researchers are familiar with unexpected off-target results in WES or WGS, i.e., incidental findings unrelated to the disease of interest. As these findings arise more commonly in the clinic, a policy is required regarding communication to patients. For example, if genetic testing in a patient with dyslipidaemia were to incidentally pick up a mutation in the familial breast cancer $B R C A 1$ gene, what is the obligation to report it? Furthermore, hundreds to thousands of incidental variants of uncertain significance (VUSs) in many genes are picked up with both WES and WGS. ${ }^{24}$ What is the most appropriate way to evaluate and communicate these findings? A conflict of interest might arise between the researcher's or physician's 'duty to inform' at-risk patients and family members, and the individual's 'right not to know'.31,32 Furthermore, genetic testing could infringe on family privacy, since results indirectly provide information about family members. ${ }^{31}$ The American College of Medical Genetics and Genomics recommends that incidental findings for certain medical conditions should be communicated to patients. ${ }^{33}$

Genetic information might also affect eligibility for work or insurance, or could otherwise lead to 'genetic discrimination'. ${ }^{31,34}$ Legislation in many countries, including the United States and Canada, is intended to protect against genetic discrimination in the workplace, and by insurance providers. However, there is little consensus regarding who can access genetic information and for what purpose.

A commonly held misbelief is that genetic testing offers diagnostic certainty when either a positive or negative result is reported. However, genetic testing is just as prone to false positives and negatives as other diagnostic tests, although 
precise sensitivity and specificity of genetic testing often cannot be determined (e.g., due to lack of reference standards). ${ }^{34-36}$ Overestimation of the 'power' of genetics can lead to both unnecessary anxiety in the case of a false positive (e.g., when a benign VUS is labelled as pathogenic, or when two causal mutations for a homozygous trait reside on the same allele but are interpreted as compound heterozygote and thus disease-causing), or inappropriate reassurance with a false negative result (e.g., for a mutation in a gene not yet associated with the disease). ${ }^{36}$

There are also financial issues to consider. While the number of clinically validated tests available is increasing, the guidelines and indications for their use and interpretation is slower to emerge, as are standards for reimbursement by insurance companies and governments. 9,30

\section{When to test}

The case for genetic testing is strengthened when there is strong suspicion (i.e., no obvious secondary causes, a strong family history, values far outside standard reference ranges, other suggestive syndromic features, or a young patient) AND when there might be a change in management, monitoring or intervention that could affect outcomes for the patient or family members (i.e., will effect eligibility for a new drugs, would potentially lead to a different choice of therapy) $\mathrm{OR}$ if there is a strong patient desire for a definitive diagnosis (Table 3). 30,36,37

$\mathrm{FH}$ is the best documented example of monogenic dyslipidaemia. $^{35}$ It is caused predominantly by $>2000$ reported mutations in the $L D L R$ gene encoding the LDL receptor. Pathogenic mutations are found in all 18 exons and the promoter region. ${ }^{1,29,35,38-40}$ Mutations in other genes, including receptor binding defective mutations in $A P O B$ encoding apolipoprotein (apo) B and gain-of-function mutations in PCSK9, are minor contributors. ${ }^{1,29,35,38,39}$ Rare autosomal recessive mutations in $L D L R A P 1$ encoding LDL receptor adaptor protein 1 can also underlie an $\mathrm{FH}$ phenotype. $^{38}$

Criteria for testing are well-established and are sometimes suggested for anyone with an LDL-C level $>5.0 \mathrm{mmol} / \mathrm{L}$ (>90th percentile) and having at least one of the following: (1) a family member with known FH or elevated cholesterol; (2) physical findings of FH including tendon or plantar xanthomas, xanthelasmas, and corneal arcus in an individual $<45$ years or their presence in a family member; (3) premature coronary heart disease (onset $<55$ and $<65$ years in men and women, respectively) in the individual or family member, or a family member with sudden premature cardiac death; or (4) if the patient meets criteria for possible or probable FH by the Simon Broome or Dutch Lipid Clinic Network criteria. $^{21,29,38,39,41}$ If an individual is identified as $\mathbf{F H}$

\section{Table 3 Possible indications for genetic testing in dyslipidaemias}

Testing might change management

Strong clinical suspicion

Patient preference

Family planning

Early interventions available

Eligibility for new drugs

Strong family history

Other, related syndromic features mutation-positive, screening of first-degree relatives is advised. ${ }^{1,41}$

The benefits of establishing a genetic diagnosis of $\mathrm{FH}$ include the ability to intervene early to minimise the future cardiovascular disease risk. Patients with genetic $\mathrm{FH}$ are at higher risk of premature ASCVD events than individuals with similar LDL-C levels but no rare variant driver for their hypercholesterolaemia. ${ }^{42,43}$ Medications, such as statins, ezetimibe, evolocumab and alirocumab, improve and sometimes even normalise LDL-C levels in FH individuals. Observational studies suggest that early intervention in this population significantly reduces the lifetime risk of ASCVD. ${ }^{28,44,43}$ Genetic testing is recommended by several guidelines as standard for FH diagnosis; it is often more accurate and definitive than phenotypic assessment alone. ${ }^{41}$ Some guidelines suggest universal FH screening; however, this has practical and economic limits. Biochemical rather than genetic cascade screening of family members of a known FH mutation carrier can be sufficient to screen for additional affected individuals. $38,41,44,46$

There are currently no guidelines for genetic testing in other monogenic dyslipidaemias. If available, we suggest testing should be based on clinical suspicion from abnormal laboratory findings, physical findings or syndromic features, or suggestive family history. An overview of the features of genetic dyslipidaemias is reported in the article by $\mathrm{Ng}$ et al. in this issue. ${ }^{47}$

\section{GENERAL GUIDELINES FOR GENETIC TESTING: PITFALLS}

As mentioned, an apparently negative genetic test does not rule out an underlying genetic cause. For instance, a 'silent' appearing variant may actually affect splicing or epigenetic modification. There may be undetected pathogenic changes (e.g., deep within introns) that are not yet linked to the disorder. Without a gold standard reference test, the false negative rate of genetic testing is difficult to quantify and varies between conditions. ${ }^{4}$ For example, mutations are found in 60-90\% of individuals classified clinically as having 'definite FH', but only in $20-30 \%$ of patients classified as 'probable FH'. ${ }^{38,48}$

False positive results also occur. ${ }^{49}$ Many detected rare mutations are not disease-causing, even when they alter the coding sequence of a known disease-related gene. Efforts to standardise diagnostic criteria for FH mutations are in progress: an FH mutation working group has created guidelines for $L D L R$ mutation pathogenicity. ${ }^{10,39}$ This may help standardise FH diagnosis worldwide, and may be useful to replicate in other disorders. The inter-assessor concordance rate using these criteria was high, but was not $100 \% .^{10}$

Also, with WES and WGS there is risk of incidentally detecting mutations in off-target genes unrelated to the referring diagnosis. ${ }^{50}$ Furthermore, reporting risk or susceptibility mutations without available interventions could cause unnecessary anxiety and expend resources, without any compensatory benefits. Along these lines, a recent study on WGS of apparently healthy Canadians found that $\sim 25 \%$ of them had 'clinically actionable' mutations, ${ }^{51}$ although there was no definitive benefit in knowing this information; more work is required.

As mentioned, discovery of VUSs by high-throughput methods is common. ${ }^{52}$ These are often previously 
unreported mutations detected in an individual presenting with a disease that were not previously known to be pathogenic. Imputing causality bioinformatically is usually not helpful, and much work is needed to improve the clinical approach to such findings. Ultimately, these findings will likely have limited clinical utility. ${ }^{52}$

\section{IMPACT ON MANAGEMENT}

Do genetic test results affect clinical management? Some studies report that providing genetic test results to clinicians did not affect practice. ${ }^{37,53,54}$ This may also hold true for $\mathrm{FH}$ genetic testing; specific $L D L R$ mutations do not correlate consistently with disease severity or ASCVD risk. ${ }^{10,29,35}$ In a study comparing lipid clinic health care providers who either had or did not have access to genetic testing results, ${ }^{53}$ clinicians reported the general impression that current genetic methods were imperfect for FH diagnosis. They believed that $10-50 \%$ of $\mathrm{FH}$ patients who formally tested as 'mutationnegative' still had an unidentified FH mutation. ${ }^{53}$ Clinical diagnosis of FH was consistently judged as more important than DNA testing: a negative genetic test result did not rule out FH when clinical suspicion was strong. ${ }^{53}$ Many felt lipid levels alone were sufficient to guide intervention; genetic test results did not change management. ${ }^{53}$ The main impact of genetic test results would be on the family members of affected individuals, especially children who could be presymptomatically diagnosed. ${ }^{53}$ These findings might have been different had the study been conducted in the PCSK9 inhibitor era.

\section{IMPACT ON PATIENTS}

The impact of genetic testing on patients depends on several factors, including disease-specific factors, past personal experiences, the method of information delivery, and patientspecific factors. ${ }^{46}$ Some studies report a lack of retention or understanding with respect to genetic risk. For example, a study of Alzheimer's susceptibility testing found that only $27 \%$ of patients tested could accurately recall their results a year later and $23 \%$ were unable to recall any information conveyed at the time of testing. ${ }^{55}$ Qualitative studies suggest a wide range of responses to genetic information depending on the strength of the genetic findings, penetrance of the disease, whether the individual has witnessed affected family members, or whether active disease symptoms are present. $^{53,56}$

In a study of newborn FH screening, elevated cholesterol levels in FH that was diagnosed based on biochemical criteria were interpreted as being more controllable and less distressing than if $\mathrm{FH}$ was diagnosed using DNA testing. ${ }^{57}$ In adults, disclosure of FH status was reported as being no more or less important than other cardiovascular risk factors. ${ }^{57}$ One study found that patients with an FH diagnosis felt less responsible for their dyslipidaemia; they seemed to interpret their dyslipidaemia as being distinct from high cholesterol in other individuals that was perceived to be lifestylerelated. ${ }^{53,58}$ Promising results were seen in patients undergoing cardiovascular risk stratification randomised to receive information on either conventional risk factors alone or conventional risk factors plus their genetic risk profile. Individuals with high genetic risk were more likely to remain on their statins than those at low genetic risk or those who were not informed of their genetic profile. ${ }^{20}$

\section{GENERAL GUIDELINES FOR GENETIC TESTING: HOW TO COUNSEL}

Counselling regarding genetic testing encompasses two domains: medical and psychological. ${ }^{24}$ Medical counselling focusses on patient education regarding the expected health impact of the mutation, explanation of disease course and management, addressing modifiable risk, and reproductive implications. ${ }^{59}$ Psychological and emotional support are particularly important in untreatable diseases, or if there are severe health manifestations; these involve making decisions that are more personal than medical. ${ }^{59}$ Who should provide counselling surrounding a genetic test result? Should this be up to the ordering physician, a specialised genetic counsellor, the family physician or another provider? Does the reporting laboratory play a role in counselling or education? For dyslipidaemias, genetic testing is often obtained through specialised lipid clinics, so these may be the best sites to provide counselling.

\section{PRACTICAL STEPS IN CLINICAL GENETIC TESTING}

Counselling should be available throughout all stages of genetic testing: pre-testing, during sample collection, posttesting and in follow-up. Pre-test counselling should focus on the informed consent process, discussing the testing procedure itself as well as the objective of testing and the risks, benefits, limitations and consequences of testing. ${ }^{24,31,52}$ The reliability and interpretation of the test should be discussed. Possible implications of a positive test result on the patient and family members should also be reviewed, including potential social and emotional effects. ${ }^{31}$ False negative and positive detection rates should be acknowledged, as well as the potential for incidental findings and VUS. Possible psychological ramifications, such as survivor or parental guilt, should be discussed. ${ }^{24}$ Attempt should be made to evaluate baseline anxiety levels, perception of risk, and health attitudes and beliefs. ${ }^{24}$

During sample collection, the patient's willingness to proceed should be confirmed, the test procedure reviewed and any questions answered before obtaining the sample. Post-test counselling includes confirming that the patient still desires their result prior to disclosure. The specific findings and their impact on the patient personally as well as his/her family members should be a major focus. ${ }^{52}$ Clinical screening and management recommendations should be addressed. ${ }^{24}$ Opportunity for questions and reflection should be provided. A discussion on cascade testing should be provided if a mutation is detected. Emotional and psychological support should be provided as required. Assistance in locating and disclosing genetic test results to family members could be offered. ${ }^{24,32}$ At follow-up, many of the same principles apply. Ongoing support and information should be provided as required.

\section{CONCLUSIONS}

The potential benefits of genetic testing include the increased likelihood of a definitive diagnosis for a patient or family, opportunity to improve management and disease prevention strategies and to help advance overall knowledge and understanding of complex metabolic pathways and processes. The decision to proceed with genetic testing in a particular 
patient should focus on a global assessment of the individual that takes into account baseline suspicion of a disorder coupled with the expected benefits of the test results, and may depend strongly on individual patient values and preferences. An understanding of the limitations of current genetic testing techniques is important when deciding to proceed with testing and for proper interpretation of the results. Ultimately, genetic testing can provide a powerful and informative tool to aid in clinical diagnosis and management, as well as in advancing care strategies beyond currently available treatments, but should be used in appropriately selected patients, by clinicians who can accurately interpret the results, to achieve the greatest benefit.

Conflicts of interest and sources of funding: AJB is supported by the Clinical Investigator Program at the Schulich School of Medicine \& Dentistry, Western University, Canada, and by the Fernand Labrie Fellowship of the Canadian Society for Endocrinology. RAH is supported by the Jacob J. Wolfe Distinguished Medical Research Chair, the Edith Schulich Vinet Research Chair in Human Genetics, and the Martha G. Blackburn Chair in Cardiovascular Research. RAH has received operating grants from the Canadian Institutes of Health Research (Foundation Grant) and the Heart and Stroke Foundation of Ontario (G-15-0009214). RAH is a consultant and speakers' bureau member for Aegerion, Akcea/Ionis, Amgen, Cerenis, Gemphire, and Sanofi.

Address for correspondence: Robert Hegele MD, Robarts Research Institute, 4288A - 1151 Richmond Street No, London, Ontario, Canada N6A 5B7. E-mail: hegele@ robarts.ca

\section{References}

1. Hopkins PN. Genotype-guided diagnosis in familial hypercholesterolemia: population burden and cascade screening. Curr Opin Lipidol 2017; 28: $136-43$

2. Hegele RA, Ban MR, Cao H, et al. Targeted next-generation sequencing in monogenic dyslipidemias. Curr Opin Lipidol 2015; 26: 103-13.

3. Dron JS, Hegele RA. Polygenic influences on dyslipidemias. Curr Opin Lipidol 2018; 29: 133-43.

4. McPherson E. Genetic diagnosis and testing in clinical practice. Clin Med Res 2006; 4: 123-9.

5. Hegele RA, Berberich AJ, Ban MR, et al. Clinical and biochemical features of different molecular etiologies of familial chylomicronemia J Clin Lipidol 2018; 12: 920-927.e4.

6. Dron JS, Wang J, Berberich AJ, et al. Large-scale deletions of the ABCA1 gene in patients with hypoalphalipoproteinemia. J Lipid Res 2018; 59: 1529-35.

7. Desai AN, Jere A. Next-generation sequencing: ready for the clinics? Clin Genet 2012; 81: 503-10.

8. Chrystoja CC, Diamandis EP. Whole genome sequencing as a diagnostic test: challenges and opportunities. Clin Chem 2014; 60: 724-33.

9. Faruki H. Genomic testing: the clinical laboratory perspective. Clin Pharmacol Ther 2013; 94: 190-2.

10. Iacocca MA, Hegele RA. Recent advances in genetic testing for familial hypercholesterolemia. Expert Rev Mol Diagn 2017; 17: 641-51.

11. Johansen CT, Dubé JB, Loyzer MN, et al. LipidSeq: a next-generation clinical resequencing panel for monogenic dyslipidemias. J Lipid Res 2014: 55: 765-72

12. Dilliott AA, Farhan SMK, Ghani M, et al. Targeted next-generation sequencing and bioinformatics pipeline to evaluate genetic determinants of constitutional disease. J Vis Exp 2018; 134: 57266.

13. Kathiresan S, Willer CJ, Peloso GM, et al. Common variants at 30 loci contribute to polygenic dyslipidemia. Nat Genet 2009; 41: 56-65.

14. Demirkan A, Amin N, Isaacs A, et al. Genetic architecture of circulating lipid levels. Eur J Hum Genet 2011; 19: 813-9.

15. Khera AV, Chaffin M, Aragam KG, et al. Genome-wide polygenic scores for common diseases identify individuals with risk equivalent to monogenic mutations. Nat Genet 2018; 50: 1219-24.
16. Talmud PJ, Shah S, Whittall R, et al. Use of low-density lipoprotein cholesterol gene score to distinguish patients with polygenic and monogenic familial hypercholesterolaemia: a case-control study. Lancet 2013; 381: 1293-301.

17. Wang J, Dron JS, Ban MR, et al. Polygenic versus monogenic causes of hypercholesterolemia ascertained clinically. Arterioscler Thromb Vasc Biol 2016; 36: 2439-45.

18. Dron JS, Wang J, Low-Kam C, et al. Polygenic determinants in extremes of high-density lipoprotein cholesterol. J Lipid Res 2017; 58: $2162-70$.

19. Iacocca MA, Wang J, Dron JS, et al. Use of next-generation sequencing to detect LDLR gene copy number variation in familial hypercholesterolemia. J Lipid Res 2017; 58: 2202-9.

20. Brown SN, Jouni H, Marroush TS, et al. Effect of disclosing genetic risk for coronary heart disease on information seeking and sharing: the MIGENES study (Myocardial Infarction Genes). Circ Cardiovasc Genet 2017; 10: e001613.

21. Paynter NP, Ridker PM, Chasman DI. Are genetic tests for atherosclerosis ready for routine clinical use? Circ Res 2016; 118: 607-19.

22. Ginsburg GS, Donahue MP, Newby LK. Prospects for personalized cardiovascular medicine: the impact of genomics. J Am Coll Cardiol 2005; 46: 1615-27.

23. Mittelstrass K, Waldenberger M. DNA methylation in human lipid metabolism and related diseases. Curr Opin Lipidol 2018; 29: 116-24.

24. Sturm AC, Hershberger RE. Genetic testing in cardiovascular medicine: current landscape and future horizons. Curr Opin Cardiol 2013; 28 : $317-25$.

25. Wang J, Ban MR, Hegele RA. Multiplex ligation-dependent probe amplification of LDLR enhances molecular diagnosis of familial hypercholesterolemia. J Lipid Res 2005; 46: 366-72.

26. Zhang H. Lysosomal acid lipase and lipid metabolism: new mechanisms, new questions, and new therapies. Curr Opin Lipidol 2018; 29 $218-23$.

27. Patel SB. Recent advances in understanding the STSL locus and ABCG5/ABCG8 biology. Curr Opin Lipidol 2014; 25: 169-75.

28. Versmissen J, Oosterveer DM, Yazdanpanah M, et al. Efficacy of statins in familial hypercholesterolaemia: a long term cohort study. BMJ 2008; 337: a2423.

29. Berberich AJ, Hegele RA. The complex molecular genetics of familial hypercholesterolaemia. Nat Rev Cardiol 2018. Jul 4: (Epub ahead of print)

30. Severin F, Borry P, Cornel MC, et al. Points to consider for prioritizing clinical genetic testing services: a European consensus process oriented at accountability for reasonableness. Eur J Hum Genet 2015; 23 : 729-35.

31. Burgess MM. Beyond consent: ethical and social issues in genetic testing. Nat Rev Genet 2001; 2: 147-51.

32. Gaff CL, Clarke AJ, Atkinson P, et al. Process and outcome in communication of genetic information within families: a systematic review. Eur J Hum Genet 2007; 15: 999-1011.

33. Kalia SS, Adelman K, Bale SJ, et al. Recommendations for reporting of secondary findings in clinical exome and genome sequencing, 2016 update (ACMG SF v2.0): a policy statement of the American College of Medical Genetics and Genomics. Genet Med 2017; 19: 249-55.

34. Burke W. Clinical validity and clinical utility of genetic tests. Curr Protoc Hum Genet 2009. Chapter 9: Unit 9.15.

35. Lamberts SW, Uitterlinden AG. Genetic testing in clinical practice. Annu Rev Med 2009; 60: 431-42.

36. Kirmani S. Molecular genetic testing in endocrinology - a practical guide. Endocr Pract 2012; 18: 85-9.

37. Lerner B, Marshall N, Oishi S, et al. The value of genetic testing: beyond clinical utility. Genet Med 2017; 19: 763-71.

38. Hughes DP, Viljoen A, Wierzbicki AS. Familial hypercholesterolaemia in the era of genetic testing. Curr Cardiol Rep 2016; 18: 42.

39. Kindt I, Mata P, Knowles JW. The role of registries and genetic databases in familial hypercholesterolemia. Curr Opin Lipidol 2017; 28 $152-60$

40. Vohnout B, Gabcova D, Huckova M, et al. Genetic testing of familial hypercholesterolemia in a real clinical setting. Wien Klin Wochenschr 2016; 128: 916-21.

41. Ademi Z, Watts GF, Pang J, et al. Cascade screening based on genetic testing is cost-effective: evidence for the implementation of models of care for familial hypercholesterolemia. J Clin Lipidol 2014; 8: 390-400.

42. Khera AV, Won HH, Peloso GM, et al. Diagnostic yield and clinical utility of sequencing familial hypercholesterolemia genes in patients with severe hypercholesterolemia. J Am Coll Cardiol 2016; 67: $2578-89$.

43. Descamps OS, Gilbeau JP, Luwaert R, et al. Impact of genetic defects on coronary atherosclerosis in patients suspected of having familial hypercholesterolaemia. Eur J Clin Invest 2003; 3: 1-9. 
44. Trent RJ, Williamson R, Sutherland GR. The "new genetics" and clinical practice. Med J Aust 2003; 178: 406-9.

45. Wiegman A, Gidding SS, Watts GF, et al. Familial hypercholesterolaemia in children and adolescents: gaining decades of life by optimizing detection and treatment. Eur Heart J 2015; 36: 2425-37.

46. Hardcastle SJ, Legge E, Laundy CS, et al. Patients' perceptions and experiences of familial hypercholesterolemia, cascade genetic screening and treatment. Int J Behav Med 2015; 22: 92-100.

47. Ng DM, Burnett JR, Bell DA, et al. Update on the diagnosis, treatment and management of rare genetic lipid disorders. Pathology 2019; 51: $193-201$.

48. Nherera L, Marks D, Minhas R, et al. Probabilistic cost-effectiveness analysis of cascade screening for familial hypercholesterolaemia using alternative diagnostic and identification strategies. Heart 2011; 97: 1175-81.

49. Douglas PS, Ginsburg GS. Clinical genomic testing: getting it right J Cardiovasc Transl Res 2008; 1: 17-20.

50. Green RC, Berg JS, Grody WW, et al. ACMG recommendations for reporting of incidental findings in clinical exome and genome sequencing. Genet Med 2013; 15: 565-74.

51. Reuter MS, Walker S, Thiruvahindrapuram B, et al. The Personal Genome Project Canada: findings from whole genome sequences of the inaugural 56 participants. CMAJ 2018; 190: E126-36.
52. Cowan J, Morales A, Dagua J, et al. Genetic testing and genetic counseling in cardiovascular genetic medicine: overview and preliminary recommendations. Congest Heart Fail 2008; 14: 97-105.

53. Will CM, Armstrong D, Marteau TM. Genetic unexceptionalism: clinician accounts of genetic testing for familial hypercholesterolaemia. Soc Sci Med 2010; 71: 910-7.

54. Yoon PW, Chen B, Faucett A, et al. Public health impact of genetic tests at the end of the 20th century. Genet Med 2001; 3: 405-10.

55. Chilibeck G, Lock M, Sehdev M. Postgenomics, uncertain futures, and the familiarization of susceptibility genes. Soc Sci Med 2011; 72 $1768-75$.

56. Claassen L, Henneman L, van der Weijden T, et al. Being at risk for cardiovascular disease: perceptions and preventive behavior in people with and without a known genetic predisposition. Psychol Health Med 2012; 17: 511-21.

57. Senior V, Smith JA, Michie S, et al. Making sense of risk: an interpretative phenomenological analysis of vulnerability to heart disease. J Health Psychol 2002; 7: 157-68.

58. Weiner K, Durrington PN. Patients' understandings and experiences of familial hypercholesterolemia. Community Genet 2008; 11: 273-82.

59. Pagon RA. Genetic testing: when to test, when to refer. Am Fam Physician 2005; 72: 33-4 